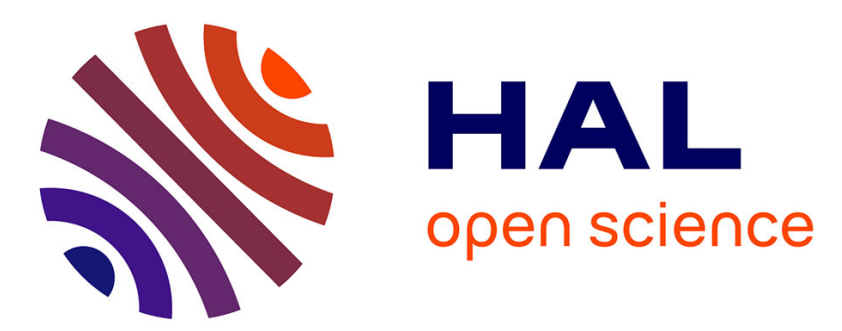

\title{
Impact of Temporal Features of Cattle Exchanges on the Size and Speed of Epidemic Outbreaks
}

\author{
Aurore Payen, Lionel Tabourier, Matthieu Latapy
}

\section{To cite this version:}

Aurore Payen, Lionel Tabourier, Matthieu Latapy. Impact of Temporal Features of Cattle Exchanges on the Size and Speed of Epidemic Outbreaks. ICCSA 2017- Workshop Agricultural and Environmental Big Data Analytics, Jul 2017, Trieste, Italy. hal-01550334

\section{HAL Id: hal-01550334 \\ https://hal.science/hal-01550334}

Submitted on 29 Jun 2017

HAL is a multi-disciplinary open access archive for the deposit and dissemination of scientific research documents, whether they are published or not. The documents may come from teaching and research institutions in France or abroad, or from public or private research centers.
L'archive ouverte pluridisciplinaire HAL, est destinée au dépôt et à la diffusion de documents scientifiques de niveau recherche, publiés ou non, émanant des établissements d'enseignement et de recherche français ou étrangers, des laboratoires publics ou privés. 


\title{
Impact of Temporal Features of Cattle Exchanges on the Size and Speed of Epidemic Outbreaks
}

\author{
Aurore Payen ${ }^{1,2}$, Lionel Tabourier ${ }^{2}$, and Matthieu Latapy ${ }^{2}$ \\ 1 AgroParisTech, Paris, France \\ 2 Sorbonne Universités, UPMC Univ Paris 06, CNRS, LIP6 UMR 7606, Paris, France \\ 凶aurore.payen@lip6.fr, lionel.tabourier@lip6.fr, matthieu.latapy@lip6.fr
}

\begin{abstract}
Databases recording cattle exchanges offer unique opportunities for a better understanding and fighting of disease spreading. Most studies model contacts with (sequences of) networks, but this approach neglects important dynamical features of exchanges, that are known to play a key role in spreading. We use here a fully dynamic modeling of contacts and empirically compare the spreading outbreaks obtained with it to the ones obtained with network approaches. We show that neglecting time information leads to significant over-estimates of actual sizes of spreading cascades, and that these sizes are much more heterogeneous than generally assumed. Our approach also makes it possible to study the speed of spreading, and we show that the observed speeds vary greatly, even for a same cascade size.
\end{abstract}

\section{Introduction}

Production of dairy and meat products is a major economic field in France. Early detection of disease outbreaks is thus a key issue for the protection of economic interests, as well as animal welfare. Among the various routes to infect holdings, such as contamination by wildlife or contacts between different herds in pastures, cattle trade movements spread pathogens at national and international level, and are thus a major way of infection.

At least three approaches can be used to study epidemic spreading occurring because of these exchanges: agent-based models, generating functions, and network approaches. The first two approaches require assumptions either on the agent behavior or about the exchanges, such as statistical equivalence of nodes of same degree for Degree-Based Mean-field theory [15] or a local treelike structure for generating functions [13,15]. In this work, we would like to study the cattle trade movements without making any assumption about the nodes' behavior and the structure of the network. That is why using network modeling seems more appropriate, as it does not require any assumption about the dynamics of exchanges.

Thanks to the creation of databases recording animal exchanges, modeling cattle trade movements for animal health purposes has started developing. Since the Bovine spongiform encephalopathy crisis of 1996, each state of the European 
Union has an obligation to identify every farm animal on its territory and to register cattle trade movements. The Base de Données Nationale d'Identification (BDNI) database is the French enforcement of this decision. The year 2005 has been described in [17], and [8] described in details years 2005 to 2009.

When studying cattle trade movements, data are often aggregated in a static network, where an edge links two holdings if at least one exchange occurred, no matter how many times and when they interact. Yet, advances in the field of temporal networks show the importance of considering the temporality of interactions among nodes to describe propagation phenomena, especially when the edges are not stable through time. Indeed, the order and the frequency of interactions are of great importance to estimate the final number of reached nodes [19].

Consequently, many recent studies also use sequences of static graphs (sequence of snapshots) to observe the evolution of networks over time $[5,8,12,14]$. For instance, [8] mainly focused on describing aggregated data for different time windows: the authors study monthly, quarterly and yearly networks. They measure the stability of several static features over successive snapshots, and also used dynamical measures, such as the reachability ratio, for which we express interest in the following. A significant asset of sequences of snapshots is that they allow the use of graph theory. However, despite the fact that each snapshot represents the aggregation of interactions that take place in a given time window, the precise dates of interactions are lost, and with them, their order and frequency. One might wonder if using a temporal network model, where the date of occurrence of a link is specified [9], could improve the evaluation of the potential size of an infection.

To answer this question, we study the French cattle trade network during the year 2005, by transforming it into a static network, sequences of monthly or quarterly networks, and a model of temporal network called link stream. After describing the network basic properties and how to measure the potential infection size of a disease in a static and a dynamical context, we compare the estimations of potential sizes of disease outbreaks, obtained using the different data representations. Finally, we propose a refinement not only to measure an outbreak size but also to take into account its propagation speed. Our contribution is then mainly methodological, comparing outbreak sizes estimations with static and temporal graph models, and proposing an intrinsically temporal measure to enhance our understanding of the measure.

\section{Dataset}

Our work relies on a data export from the BDNI which contains all cattle trade movements from 2005 to 2015 inside France. This makes approximately 148 millions cattle transfers in the following format: date of the transfer, origin holding, destination holding, animal identification number. We do not use animal identification numbers here, and focus on the fact that an exchange occurred between two given holdings at a given date. 
In this paper, we focus on year 2005 because it already received much attention in previous works $[8,17]$. This choice makes it easier to compare our results to existing ones, and we checked that they still hold for recent years. We therefore consider 2005 as representative of all years, to this regard. In this subsection, we only present here a few basic properties of the dataset that help us analyze the measurements implemented in the rest of this work.

During 2005, 245,821 holdings exchanged at least one animal with another holding, leading to a collection of approximately 10 million cattle transfers. We display in Fig. 1 the distributions of the number of outgoing and incoming movements for each holding. We also show in Fig. 2 (left) the total number of transfers occurring per week during the whole year, and in Fig. 2 (right) the total number of transfers occurring per day, for several weeks.
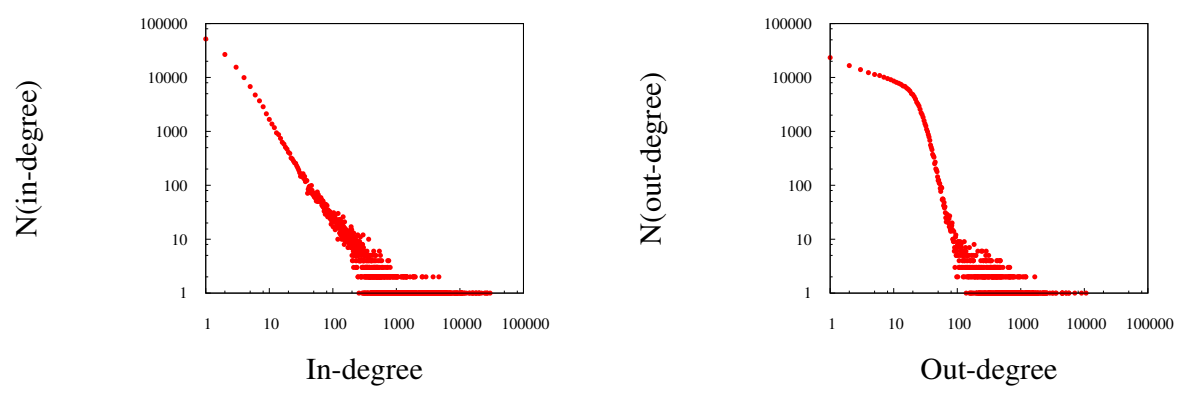

Figure 1. Left: distribution of incoming movements per holding in 2005, in log-log scales. For each value $x$ on the horizontal axis, we plot the number of holdings that were the destination of $x$ cattle movement during 2005. Right: distribution of outgoing movements per holding in 2005 .
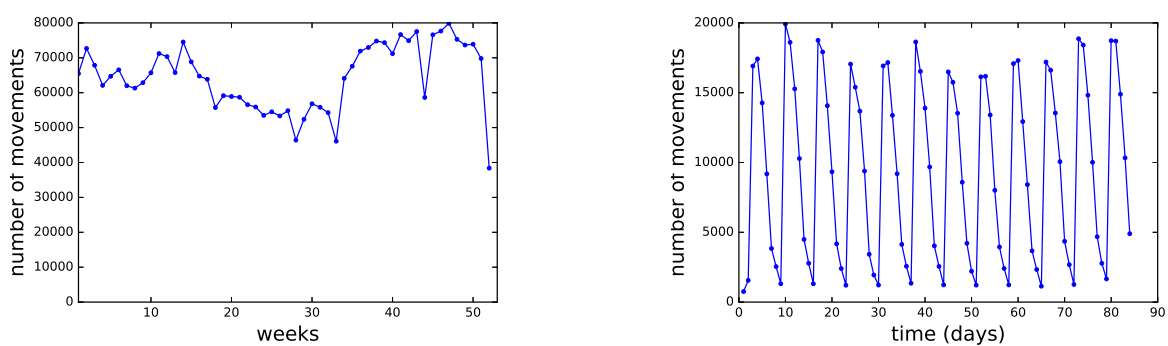

Figure 2. Left: weekly number of transfers in the database in 2005. Right: daily number of transfers in the database for the first 12 weeks of 2005. 
The movement distributions clearly confirm the heterogeneity between holdings, already observed in previous works $[8,17]$ : although some holdings are the origin or destination of up to approximately one thousand in- or out-going movements, the vast majority of holdings are involved in only a few movements, with all intermediary behaviors present in the data. Interestingly, there is a cutoff in the out-going movement distribution indicating that holdings involved in more than 100 out-going movements are exceptions (less than $2 \%$ of the nodes).

The number of movements per day (Fig. 2, right) is also interesting in the context of investigating the dynamical properties of the dataset. As already discussed in previous works on different animal trade networks [3,6,11], it shows that the short-term activity strongly changes with time, with peaks of activity on Mondays that progressively vanish through the week. Such variations may have an important impact on spreading phenomena.

It has also been observed that the French cattle trade network is asymmetric [8], in the sense that the existence of a link from $i$ to $j$ does not systematically involve the existence of a link from $j$ to $i$. This property may be measured using the reciprocity ratio, which is the fraction of reciprocated links during a given period. We measure that this ratio is indeed 0.52 for the yearly network, and of the same order of magnitude for monthly networks (from 0.51 to 0.53 ). It stems from the fact that most of the holdings sell or transfer animals to other holdings, while a much lower amount receive or buy animals. This property also has direct consequences on the potential outbreak sizes, that will be discussed later.

\section{Data Modeling}

The data presented above may be represented in several ways. In spreading phenomena studies, the main approach consists in modeling relations between holdings using networks (i.e., sets of nodes and links between them), which capture the structure of exchanges. The direction of exchanges plays a key role in spreading phenomena, as a cattle transfer from holding $A$ to holding $B$ is different from a transfer from $B$ to $A$, to this regard. Likewise, the time-ordering of cattle transfers is important: a transfer from $A$ to $B$ followed by a transfer from $B$ to $C$ is different from a transfer from $B$ to $C$ followed by a transfer from $A$ to $B$, regarding spreading.

After presenting how the problem of estimating the potential size of an outbreak is usually addressed in the static and snapshots case, we propose an adaptation to link streams, a dynamical model of networks. In all cases, we consider directed links. Previous studies already insisted on these temporal aspects $[7,14,18]$, and introduced notions such as the in/outgoing infection chains in order to better capture temporal features of cattle transfers. Our approach is related to these ones, focusing on the comparison that can be made with the snapshot case.

The most basic approach ignores temporal information: nodes represent holdings and there is a directed link from $i$ to $j$ if there was at least one cattle transfer from holding $i$ to holding $j$ in 2005. We call this model the static network. 
On 2005 dataset, it has 245,821 nodes and 1,646,510 directed links. Its degree distributions are the quantities plotted in Fig. 1.

In order to take into account the temporal features of exchanges while still using network formalisms, one may divide the data into time slices, each slice corresponding to a static network called snapshot. This leads to sequences of snapshots, each capturing the structure of exchanges during a time slice. Choosing appropriate time slices is a difficult question in general, but monthly and quarterly time slices are generally used for cattle trade exchanges, leading to 12 and 4 snapshots respectively, see for instance [8]. Choosing small time slices leads to a better conservation of the dynamics but also to snapshots with less information, while choosing larger time slices gives more complex snapshots but the dynamics within each time slice is lost.

In our case, the number of active nodes, that is to say holdings involved in at least one exchange, varies from 105295 (43\% of the total) to $138907(57 \%)$ per month (see Fig. 3), or from 180930 (74\%) to 196166 (80\%) quarterly.

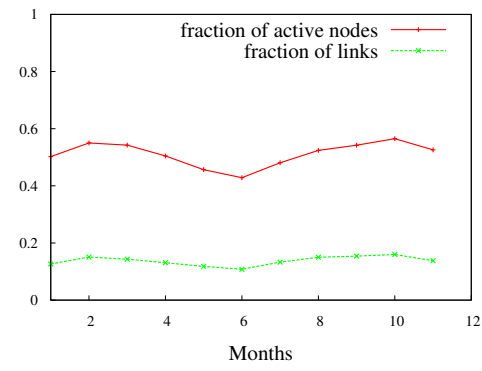

Figure 3. Fraction of active nodes and links for each monthly network

Finally, in order to capture all the dynamics in the data, one may use the link stream or temporal network formalisms $[10,20]$. A link stream is a sequence of triplets $(t, i, j)$ indicating that an interaction (here at least one cattle transfer) occurred at time $t$ from node $i$ to node $j$ (here holdings). In our case, the obtained link stream involves 245,821 nodes and 3,355,680 temporal links.

\section{Infection Modeling}

Accurate modeling of infections is a challenge in itself [16]. However, most models rely on the assumption that infections spread over a population through the links of a network representing contacts between individuals. This leads in particular to the Susceptible-Infected (SI) model, which represents a worst case scenario: the disease spreads from a node $A$ to all its neighbors as soon as $A$ is infected, and nodes never recover. The size and speed of obtained spreading cascades are therefore upper bounds of what one may expect in reality. The SI model does 

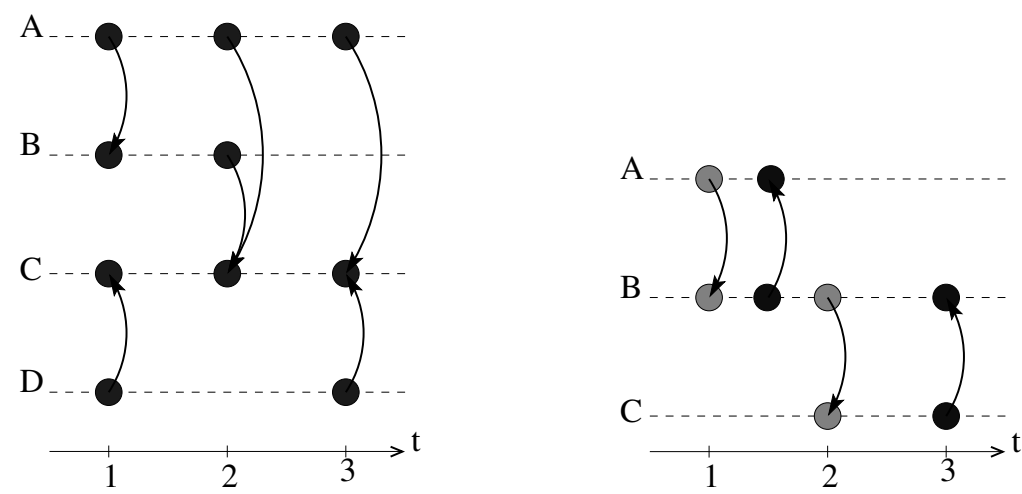

Figure 4. Left: Link stream representation of interactions, A, B, C and D are holdings and a directed link at $t$ represents a movement from the departure holding to the arrival holding at time $t$. Right: SI cascade on a link stream, starting from $\mathrm{A}$ at time 1 , and reaching nodes $\mathrm{B}$ and then $\mathrm{C}$ following directed links.

not claim to be realistic, yet, as we are interested here in quantifying the size and speed of potential outbreaks, without any assumption on the disease under concern, we consider this model as a baseline and study its behavior in static and dynamical settings.
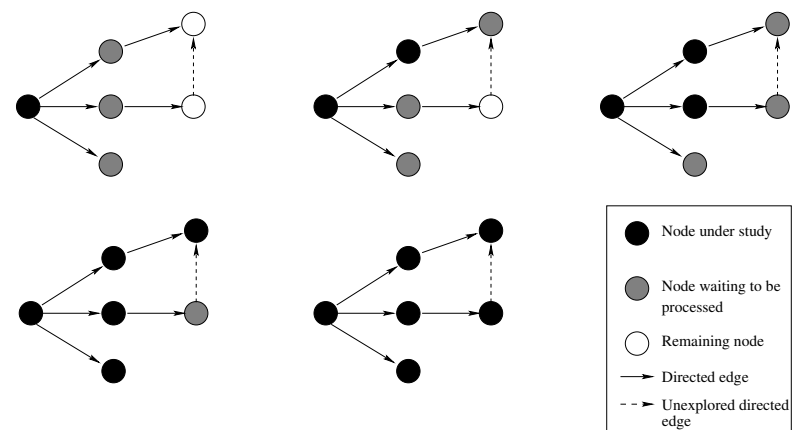

Figure 5. SI cascade in the static snapshot case starting from the leftmost node: at each step, the neighbors (gray) of an infected node (black) are reached, until all the nodes are processed.

Many studies - e.g. [1] - consider the undirected version of the static graph defined above: they consider that a link from $A$ to $B$ also induces a link from $B$ to $A$. Then, the key property for spreading phenomena is the size of the connected components. Indeed, a connected component is a maximal set of nodes such that there is a path from any node in the set to any other node in the set. As a consequence, any spreading starting in a connected component will eventually 
reach all the nodes of this connected component, and so connected component sizes give the sizes of epidemic outbreaks according to the SI model. In particular, most real-world networks have a connected component that contains most nodes of the network, called the Giant Connected Component, or GCC. Using such a representation, most epidemic outbreaks therefore reach all the nodes in the GCC, i.e. most nodes of the network.

Considering directed static networks is more realistic and leads to more detailed insights. The connected components of the undirected network are then called the Weakly Connected Components (WCC) of the directed network, and a Strongly Connected Component (SCC) is a set of nodes such that there is a directed path from any node in the set to any other node in the set. Strongly connected components are therefore included in weakly connected components, and an infection starting in a weakly connected component will not in general reach all nodes of this component. Instead, an infection starting in a strongly connected component will spread in the whole strongly connected component. Therefore, the size of strongly connected components, and in particular the size of the largest one, are key features for epidemic studies [17]. However, an infection starting in a strongly connected component will also spread to all nodes reachable from it, which are not necessarily in the component. Likewise, some infections starting outside a strongly connected component may reach it and all its nodes. Therefore, considering connected components misses much information.

As a consequence, we decided to conduct our study by truly simulating SI spreading phenomena: we start from a given node $A$ and compute the set of all nodes reachable from $A$; as this would be the set of nodes reached by a SI epidemic spreading starting at $A$, we call it a $S I$ cascade. As we will see, such cascades are strongly related to (weakly and strongly) connected components in some situations, but they allow for a more precise understanding of spreading phenomena in other situations.

More precisely, in the case of the static directed network modeling of cattle mobility, we compute the size of the SI cascade starting from any node. In the case of snapshot modeling, we compute these sizes for each snapshot (each node therefore leads to 12 cascades in monthly snapshots). In the case of link streams modeling, the situation is more subtle: the SI spreading follows links in their temporal order, see Fig. 4 (right). In particular, if links from $A$ to $B$ and from $B$ to $A$ are followed by links from $B$ to $C$ and from $C$ to $B$ then a disease may spread from $A$ to $B$ and then to $C$ but not the converse (although the directed links from $C$ to $B$ and from $B$ to $A$ do exist, they are not in the appropriate temporal order). In this setting, SI cascades are therefore constrained not only by link directions but also by time ordering. Equivalent objects were previously defined and used in the literature, they are called outgoing infection chains in [21] or in [7].

In order to compare SI cascades in link streams to the ones in snapshots, we add the following constraint: for a given time duration (one or three months in general), we consider only the nodes reached within this duration after the cascade starts. Indeed, cascades in snapshots represent epidemics that have a 
limited time to spread; adding this time limit to spreadings in link streams makes the comparison fair. In addition, we have to choose a starting time in the link stream context. In our experiments, we choose for each node a random time and then observe SI cascades starting from each node at this time.

In terms of computational complexity, we can compare the cascading process on link streams to the one on static graphs. Setting a propagation duration have an impact on the time complexity of calculations. Let $\mathscr{L}$ be the set of temporal links $(t, i, j)$, with t between the departure time and the propagation duration. The time complexity is $\mathcal{O}(n|\mathscr{L}|)$, with $n$ the number of nodes in the network. It only depends on the propagation duration and not on the total duration of the dataset. In the static case, it depends on the length of snapshots and the number of active nodes and links in this period, so the time complexity is smaller than $\mathcal{O}(n m)$, with $m$ the total number of links of the network. Experimentally we observed that the computation of SI cascades is faster in the sequence of snapshots case than in the link stream case on a standard workstation: results on link streams are obtained in a couple of days, while some minutes to a few hours are enough on sequences of snapshots. In both cases, it is possible to make a sample of source nodes to speed up the calculations, but it raises the question of representativity of the results. Concerning memory complexity, computing SI cascades is in $\mathcal{O}(n)$, as it only requires to store their state (susceptible or infectious) over time. The link stream is read link by link in increasing value of time, to update the infectious state of the nodes. Thus, it is not needed to load it in memory, contrary to sequences of snapshots. The required memory space is then smaller in the case of link stream.

\section{Size of Cascades}

This section is devoted to the study of cascade sizes (i.e. the total number of nodes reached by SI cascades) in the frameworks described above. Our goal is to gain insight on the effect of temporal features of cattle mobility on cascade sizes.
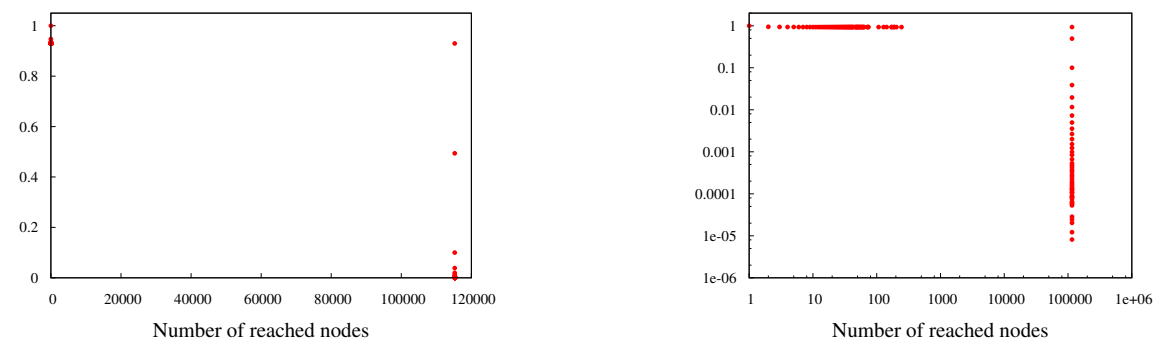

Figure 6. Inverse cumulative distribution of cascades sizes for the 2005 static directed network. Left: lin-lin scales. Right: log-log scales. 
Let us first consider the static directed network. The distribution of cascade sizes is given in Fig. 6. It clearly displays two different situations: either cascade sizes are over 100,000 nodes (93\% of them reach more than $41 \%$ of active nodes), or they are much smaller, between 1 and 300 nodes. This is due to the fact that the network has the structure depicted in Fig. 7. According to this representation, commonly used as a large-scale map of the world wide web [4], the nodes are spread over different groups: a central core, the GSCC, where any node can reach any other node following a directed path; the IN component, where some nodes can reach nodes of the GSCC following a directed path, but cannot be reached by the nodes of the GSCC. Reciprocally, some nodes can be reached by the nodes of the GSCC following a directed path, but cannot reach it, they are located downstream to it, and constitute the OUT component. The remaining nodes are either isolated in small connected components, or part of structures called tendrils and tubes. Tendrils are subgraphs going either out from the in-component without reaching the GSCC, or subgraphs going into the outcomponent without coming from the GSCC. Tubes connect directly the in- to the out-component, without going through the GSCC.

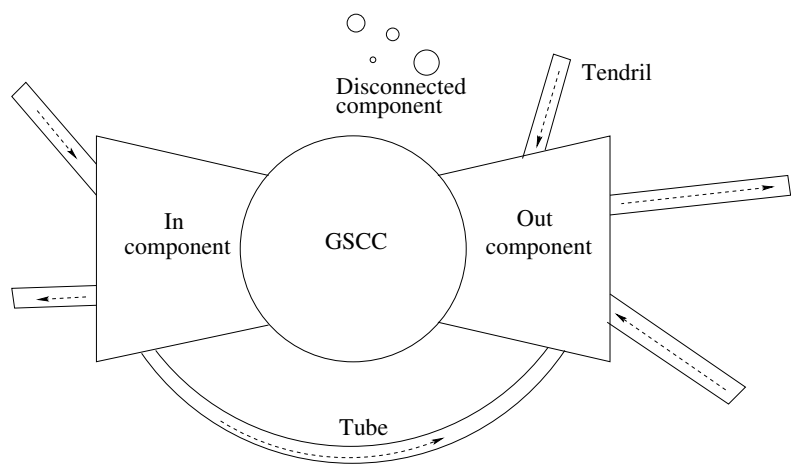

Figure 7. Bow-tie structure of a directed graph with a giant strongly connected component.

Any cascade starting in the largest strongly connected component will reach all this component ( $44 \%$ of all nodes) and the OUT component ( $3 \%$ of all nodes). In our case, this makes a total of 115,250 nodes. All cascades starting in the IN component will also reach these nodes, and a few others: the part of the IN component between them and the strongly connected component, as well as few other nodes. All these cascades therefore have very similar sizes, and reach a large fraction of all nodes in the network. Finally, a few cascades start in the OUT component, or at other nodes that do not reach the largest strongly connected component ( $7 \%$ of them), they reach only a small part of the network and thus lead to much smaller cascade sizes. These results are consistent with previous results on the size of giant connected components, and confirm that taking into 
account the direction of links is crucial: SI cascades would otherwise reach $99 \%$ of nodes [7,17]. Considering the distribution of cascade sizes however highlights the fact that two very focused regimes co-exist, and that other cascade sizes never occur.

Now, we display in Fig. 8 the cascade size distribution for the monthly snapshots and the corresponding experiments for the link stream modeling. Quarterly snapshots give very similar results so we do not plot them here. Similarly to the static case, the cascade size distribution obtained for monthly snapshots display two distinct regimes, and for the same reason: each snapshot has a bow-tie largescale shape. However, the largest cascades are much smaller than in the static network: they only reach $8 \%$ (July) to $13 \%$ (April) of nodes. This is also true for quarterly snapshots, in which cascades reach at most $23 \%$ of nodes. This may be due to the fact that spreading time is bounded, but it also shows that the cattle mobility is far from being a monthly or quarterly repetition of movements similar to the ones performed at the yearly level. This confirms the fact that time information is crucial to have an estimate of the potential epidemic outbreak sizes, as pointed out previously by several works $[1,7,19]$. It is the main motivation for turning to link stream modeling, that captures this information much more precisely.
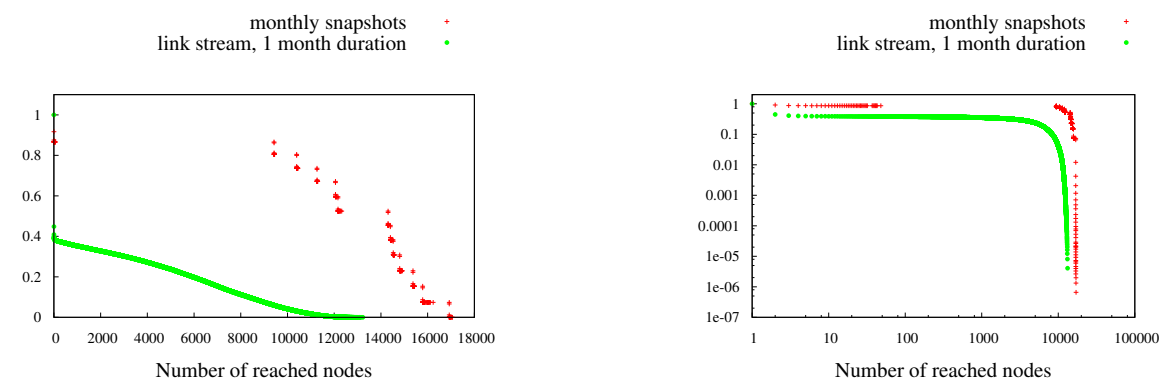

Figure 8. Inverse cumulative distribution of cascades sizes for the 2005 monthly snapshot networks (in green, x points) and for the link stream modeling (in red, + points). Left: lin-lin scales. Right: log-log scales. The vertical axis is normalized to account for the fact that different numbers of cascades are considered in the two cases.

The results using link stream modeling first show that maximal SI cascades lasting one month actually reach $5 \%$ (i.e. 13225$)$ of all nodes only ( $13 \%$ with a 3 months propagation duration). This confirms that neglecting temporal information leads to significant over-estimates of the possible size of epidemic outbreaks. The distribution even shows that the number of negligible SI cascades is much larger than indicated by snapshots: respectively $60 \%$ of SI cascades are of size smaller than 5 nodes in the link stream, compared to $13 \%$ in monthly snapshots.

Fig. 8 also displays another striking fact: whereas static network, quarterly snapshot and monthly snapshot modelings consistently lead to two different 
kinds of SI cascades (a few very small ones and a huge majority reaching a large part of the network, with nothing in between), link streams lead to a continuum in the observed cascade sizes. Although many cascades are large and many are very small, there are also all kinds of cascade sizes in between, and the heterogeneity of cascade sizes is much higher.

\section{$6 \quad$ Speed of Cascades}

One key advantage of the link stream modeling is that it accounts for the speed of spreading, and not only for the number of nodes eventually reached, which is a key feature for fighting epidemic outbreaks. We therefore dedicate this section to the study of the speed of SI cascades in link streams.
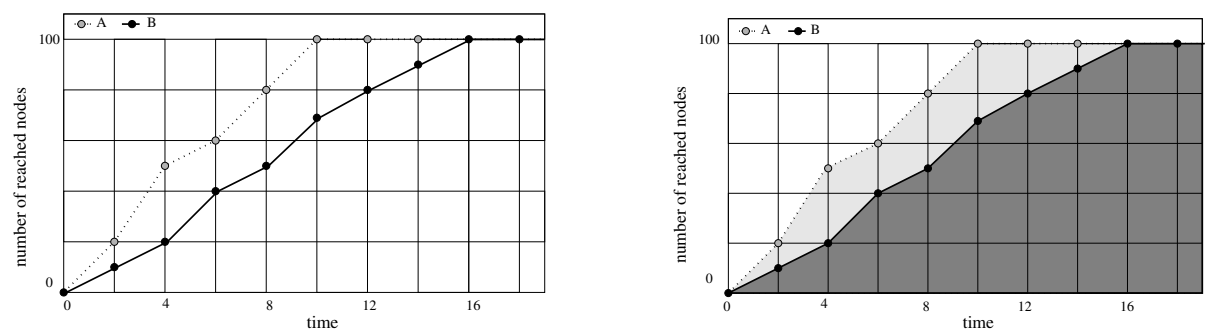

Figure 9. Left: Number of reached nodes over time by cascades from two different source nodes (A and B). Right: Visualization of the virulence (area under the curve of the number of reached nodes over time).

In Fig. 9 (left), the SI cascades from $\mathrm{A}$ and $\mathrm{B}$ reach the same number of nodes at the end of the propagation period. Yet, the number of nodes reached at a given time is higher for the propagation from source A than from source B. As a consequence, if the cascade represents the spreading of a disease, the first situation certainly implies a higher level of risk than the second one. Measuring the number of reached nodes does not allow to make this distinction. That is why we also calculated what we called the virulence of a cascade, that is simply the area under the curve of the number of reached nodes over time (Fig. 9, right), using the following definition. Let $t_{i}$ be the beginning of the spreading, $d$ the propagation duration (parameter of the model), and $n(t)$ the number of reached nodes at time $t$ :

$$
\text { virulence }=\sum_{t=t_{i}}^{t_{i}+d} n(t)
$$

Using this measure, the SI cascade starting from B gets a lower virulence score than the spreading starting from A. It allows us to differentiate the case described in Fig. 9 (left), where two cascades reach the same number of nodes with different speeds. 
We plot in Fig. 10 the virulence as a function of the final number of reached nodes. We observe that for a given number of infected nodes, the corresponding scores of virulence cover a broad range of values, especially for large spreading cascades. For instance, for a score of 6000 reached nodes, the virulence spreads from about 25000 to about 75000 , that is to say three times as much. This means that cascades of the same size may correspond to very different virulences, in other words very different propagation speeds. Similar observations can be made with a propagation duration of 3 months. Refining the comparison on the number of reached nodes and the virulence and their impact on the propagation sizes is a perspective of this study.

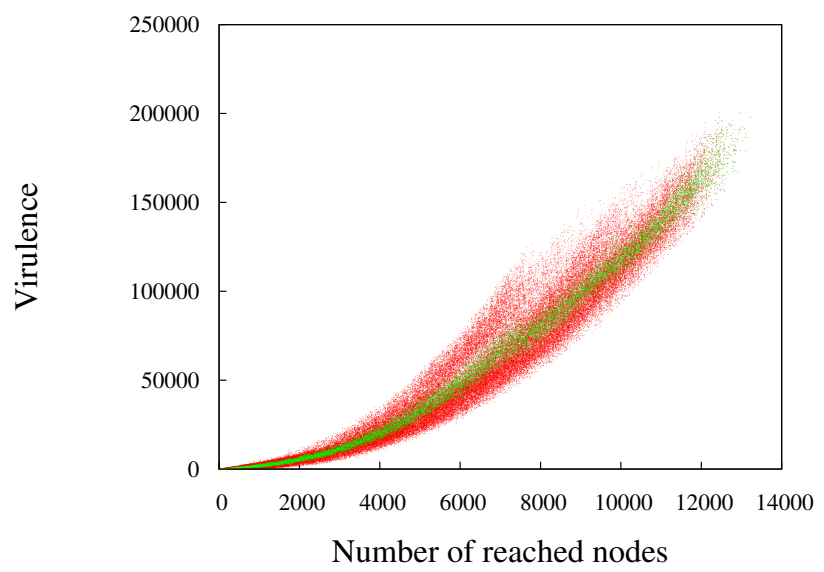

Figure 10. Virulence as a function of the final number of reached nodes. In green, average virulence values for a fixed number of reached nodes.

\section{Conclusion}

In this study, we evaluate the potential epidemic outbreak sizes, by taking into account temporal information in a network-based representation of cattle trade movement. For this purpose, we used data extracted from the year 2005 of the French database of cattle trade movements, and compared the sizes of SI cascades as produced using snapshot-based representations, to the closest possible protocol on a link stream representation of the data. We observed that cascades on static networks are systematically larger than the ones on link streams and thus that not taking into account the temporality of contacts leads to overestimating potential infection sizes. Moreover, while the cascades on static snapshots display a clear 2-mode behavior, where cascades are either very small or close to the maximum size, cascades on link streams can have any kind of size between these two extremes. Besides that, using SI cascades on link stream offers 
the possibility to take into account the spreading speeds, and not only the total number of reached nodes. Our results show that a same number of infected nodes can correspond to a wide range of propagation speed, which suggests that our measure should be a better tool to estimate the impact of an outbreak.

This study leads to several relevant considerations for future work. In the $B D N I$ dataset, information about the type of holding (farms, markets, assembling centers...) is available. A special focus on the proportion of holdings being reached by SI cascades depending on their type would be interesting, in order to gain insight on whether the category they belong to leads to different risk exposures to disease outbreak.

In the context of controlling pathogen spread, targeted control is a key issue. One of the common procedures to test an intervention strategy is to measure the size of the GSCC depending on the number of removed nodes, chosen according to the strategy. For instance, some authors measured the reduction in size of the giant connected component as a function of the number of removed node, chosen in decreasing order of their number of connections, strength, or centrality measurements [2,8], while others focused on the type of holdings removed (assembling centers, markets, farms, etc.) [17]. Taking into account the temporality of interactions allows to distinguish various intermediate cascades sizes between the two extremes (very small sizes or very close to the maximal size). Therefore, it is expected that using the cascade size distribution would be a more precise assessment of the impact of the different control strategies than a GSCC-based analysis. Moreover, nodes are targeted according to their features in static snapshots, while [1] warned that using past snapshots information to devise efficient control strategies in the present might be inefficient. That is why taking advantage of link stream temporal information and adapting strategies to focus on dynamical features in order to select the key nodes to be removed is also a perspective of great interest.

Finally, we only considered the cattle trade movements as propagation routes. Thanks to the location of holdings, infection by contact in pastures may be modeled by adding links between holdings in the same neighborhood. Providing

that we circumvent privacy issues related to the geographical location, such an approach would allow a more accurate modeling of contacts, but would also complexify the studied network.

\section{Acknowledgements}

This work is funded in part by the European Commission H2020 FETPROACT 2016-2017 program under grant 732942 (ODYCCEUS), by the ANR (French National Agency of Research) under grants ANR-15-CE38-0001 (AlgoDiv) and ANR-13-CORD-0017-01 (CODDDE), by the French program "PIA - Usages, services et contenus innovants" under grant O18062-44430 (REQUEST), and by the Ile-de-France program FUI21 under grant 16010629 (iTRAC). 


\section{References}

1. Bajardi, P., Barrat, A., Natale, F., Savini, L., Colizza, V.: Dynamical patterns of cattle trade movements. PLoS ONE 6(5) (2011)

2. Bajardi, P., Barrat, A., Savini, L., Colizza, V.: Optimizing surveillance for livestock disease spreading through animal movements. Journal of The Royal Society Interface 9(76), 2814-2825 (2012)

3. Belik, V., Fiebig, F., Lentz, H.H.K., Hövel, P.: Controlling contagious processes on temporal networks via adaptive rewiring (2015)

4. Broder, A., Kumar, R., Maghoul, F., Raghavan, P., Rajagopalan, S., Stata, R., Tomkins, A., Wiener, J.: Graph structure in the web. Computer networks 33(1), 309-320 (2000)

5. Büttner, K., Krieter, J., Traulsen, A., Traulsen, I.: Efficient Interruption of Infection Chains by Targeted Removal of Central Holdings in an Animal Trade Network. PLoS ONE 8(9) (2013)

6. Büttner, K., Salau, J., Krieter, J.: Quality assessment of static aggregation compared to the temporal approach based on a pig trade network in Northern Germany. Preventive Veterinary Medicine 129, 1-8 (2016)

7. Dube, C., Ribble, C., Kelton, D., Mcnab, B.: Comparing Network Analysis Measures to Determine Potential Epidemic Size of Highly Contagious Exotic Diseases in Fragmented Monthly Networks of Dairy Cattle Movements in Ontario, Canada 55, 382-392 (2008)

8. Dutta, B.L., Ezanno, P., Vergu, E.: Characteristics of the spatio-temporal network of cattle movements in France over a 5-year period. Preventive Veterinary Medicine 117(1), 79-94 (2014)

9. Holme, P., Liljeros, F.: Birth and death of links control disease spreading in empirical contact networks. Scientific reports 4, 4999 (2014)

10. Holme, P., Saramäki, J.: Temporal networks. Physics reports 519(3), 97-125 (2012)

11. Lentz, H.H.K., Selhorst, T., Sokolov, I.M.: Unfolding Accessibility Provides a Macroscopic Approach to Temporal Networks. Physical Review Letters 110(11), $1-5(2013)$

12. Natale, F., Giovannini, A., Savini, L., Palma, D., Possenti, L., Fiore, G., Calistri, P.: Network analysis of Italian cattle trade patterns and evaluation of risks for potential disease spread. Preventive Veterinary Medicine 92(4), 341-350 (2009)

13. Newman, M.E.J.: Spread of epidemic disease on networks. Physical Review E Statistical, Nonlinear, and Soft Matter Physics 66(1), 1-11 (2002)

14. Nöremark, M., Hakansson, N., Lewerin, S.S., Lindberg, A., Jonsson, A.: Network analysis of cattle and pig movements in Sweden: Measures relevant for disease control and risk based surveillance. Preventive Veterinary Medicine 99(2-4), 78-90 (2011)

15. Pastor-Satorras, R., Castellano, C., Van Mieghem, P., Vespignani, A.: Epidemic processes in complex networks. Reviews of Modern Physics 87(3), 925-979 (2015)

16. Pellis, L., Ball, F., Bansal, S., Eames, K., House, T., Isham, V., Trapman, P.: Eight challenges for network epidemic models. Epidemics 10, 58-62 (2015)

17. Rautureau, S., Dufour, B., Durand, B.: Vulnerability of Animal Trade Networks to The Spread of Infectious Diseases: A Methodological Approach Applied to Evaluation and Emergency Control Strategies in Cattle, France, 2005. Transboundary and Emerging Diseases 58(2), 110-120 (2011)

18. Schärrer, S., Widgren, S., Schwermer, H., Lindberg, A., Vidondo, B., Zinsstag, J., Reist, M.: Evaluation of farm-level parameters derived from animal movements for 
use in risk-based surveillance programmes of cattle in switzerland. BMC veterinary research 11(1), 149 (2015)

19. Vernon, M.C., Keeling, M.J.: Representing the UK's cattle herd as static and dynamic networks. Proceedings. Biological sciences / The Royal Society 276(1656), 469-76 (2009)

20. Viard, J., Latapy, M.: Identifying roles in an ip network with temporal and structural density. In: Computer Communications Workshops (INFOCOM WKSHPS), 2014 IEEE Conference on. pp. 801-806. IEEE (2014)

21. Webb, C.R.: Investigating the potential spread of infectious diseases of sheep via agricultural shows in Great Britain. Epidemiology and infection 134(1), 31-40 (2006) 